\title{
Low-cost wearable Multichannel Surface EMG Acquisition for Prosthetic Hand Control
}

\author{
Davide Brunelli and Andualem Maereg Tadesse \\ Department of Industrial Engineering \\ University of Trento \\ Trento, Italy \\ \{name.surname\}@unitn.it
}

\author{
Bernhard Vodermayer, Markus Nowak and \\ Claudio Castellini \\ German Aerospace Research Center - DLR \\ Germany \\ \{name.surname\}@dlr.de
}

\begin{abstract}
Prosthetic hand control based on the acquisition and processing of surface electromyography signals (sEMG) is a well-established method that makes use of the electric potentials evoked by the physiological contraction processes of one or more muscles. Furthermore intelligent mobile medical devices are on the brink of introducing safe and highly sophisticated systems to help a broad patient community to regain a considerable amount of life quality. The major challenges which are inherent in such integrated system's design are mainly to be found in obtaining a compact system with a long mobile autonomy, capable of delivering the required signal requirements for EMG based prosthetic control with up to 32 simultaneous acquisition channels and - with an eye on a possible future exploitation as a medical device - a proper perspective on a low priced system. Therefore, according to these requirements we present a wireless, mobile platform for acquisition and communication of SEMG signals embedded into a complete mobile control system structure. This environment further includes a portable device such as a laptop providing the necessary computational power for the control and a commercially available robotic handprosthesis. Means of communication among those devices are based on the Bluetooth standard. We show, that the developed low cost mobile device can be used for proper prosthesis control and that the device can rely on a continuous operation for the usual daily life usage of a patient.
\end{abstract}

Keywords-EMG, wireless communication, Bluetooth.

\section{INTRODUCTION}

In recent years, prosthetic technology for lower arm limb or hand amputated patients has revealed impressive advances by evolving from simple and fixed anthropomorphic extensions to real-time actuated electronic prosthetics. The preferred signals used to control hand gesture are derived from non-invasive, so called surface electromyography (sEMG) sensors, providing broad signal information about the corresponding muscle activity [1].

These sensors incorporate capturing and amplification of evoked muscle action potentials by one or more related muscles in the patient's remaining stump. The signals are processed by detection algorithms to control a prosthetic device in accordance with the patient's intended movements such as grasping, wrist rotation or flexions. This approach tries to re- enable the natural way of controlling the lost hand by contracting the remaining corresponding muscles, thus offering a more intuitive approach in contrary to the EMG derivation of other muscle groups, e.g. in the pectoral region where patients have to train for intensive and longer sessions to regain their formerly lived quality of life [2],[3].

The promising opportunities from this technology are widely recognized by the scientific community which has started to investigate sEMG also for control commands in many other applications such as gesture-based interfaces for virtual reality games, virtual keyboards, and human computer interface etc. [4],[5].

Depending on the number of sEMG sensors and the types or groups of muscles used to feed the control algorithms, it is possible to perform different classes of hand gestures: from coarse hand grasping, wrist and hand movements, to individual finger flexions.

To achieve smooth and articulated prosthesis movements a higher number of sEMG sensors along with multi-channel signal acquisition is beneficial [6].

In this paper we address the possibility to acquire sEMG signals up to 32 channels simultaneously, providing adequate bandwidth and signal resolution for complex gesture recognition and prosthetic control. A wireless, mobile low-cost system will be introduced, that makes use of commercially available, medical device grade components of the shelf COTS, like the Otto-Bock 13E200 electrodes for sEMG signal reading or Touch Bionics' i-LIMB Ultra Revolution robotic hand prosthesis. The interconnection of these components will be presented to be completely wireless and providing an independent autonomy that renders the system useable for patients' daily life actions helping them to regain their formerly lived quality of life. Corresponding system performance will be discussed.

The paper is organized as follows: in Section II we present the state of the art of wireless prosthetic hand controlled by sEMG signals. The modeling and the design of the wireless acquisition system is presented in Section III with emphasis on the design choices for power optimization. Experimental 
measurements and discussion about performance and energy autonomy is discussed in Section IV. Finally Section V concludes the paper.

\section{RELATED WORK}

The concept of mobile medical devices including intelligent prostheses has by now a long term history. But still big challenges arise when physiologic processes have to be transformed into actions of the medical device.

With respect to multi-channel acquisition of signals evoked by the human body, Wang [7] addresses the design of a multichannel data acquisition system with 32 channels for EEG signal based application. In this field, aggressive power management techniques have been proposed in [8] by applying approximate computing paradigm to the monitoring and ECG signals.

Of course the signal requirements for EEG signals differ from those in sEMG applications, but in this case the number of signals and bandwidth are in the same range. Intensive electronic filtering is utilized by a complex analog circuitry and an on board DSP. The system is capable of acquiring up to 24 unipolar and eight bipolar electrodes. The usable signal bandwidth is $100 \mathrm{~Hz}$ realized by complex filtering. The system does not provide any mobility in the current state as a wired connection to a PC, responsible for the processing of the acquired signals.

Another application in the field by Vaca Benitez et al. [9] presents an EMG-controlled arm orthosis in an early prototype stage. The control system is fed with signals acquired from a commercially available sEMG-signal recorder Brain Amp ExG MR by the German Company Brain Products GmbH, Gilching [10]. This type of the device is available in two versions with 8 or 16 channels and is especially prepared for the use within CT or MRT scanners. Analog-to-digital conversion is carried out with a resolution of 16bit with a maximum of $5 \mathrm{kHz}$ per channel. Fiber optic communication is used due to environmental requirements, thus the device's portability is restricted to the cabling. The power consumption is $150 \mathrm{~mA}$ in active mode, yet this subsystem itself has a weight of $1.1 \mathrm{~kg}$. One PowerPack for the use together with the system with a capacity of $6500 \mathrm{mAh}$, providing approximately $40 \mathrm{~h}$ of independent operation, has a weight of $130 \mathrm{~g}$ [11]. Vaca Benitez et al. further describe, that the usable signal is obtained by filtering down to a bandwidth of $20 \mathrm{~Hz}$ and transferring it to a $\mathrm{PC}$ for further processing. Control of the orthosis will be part of upcoming scientific work.

Lee et al. [12] also comprise a portable sEMG recording system that can be mounted into a wireless environment using Bluetooth connectivity. The system provides 10 parallel channels including the complete signal chain with sensor, preamplification and initial processing, second amplification, conversion and finally the distribution via the Bluetooth interface to a processing station. Lee et al. report above that to have reached a wireless maximum data rate of $723 \mathrm{kbps}$ while gaining a sample rate of $2000 \mathrm{~Hz}$ for ten simultaneous channels acquired. The power consumption of the Bluetooth device is described to be $25 \mathrm{~mA}$. However, the total power consumption and autonomy depend also on the acquisition part.

A multichannel sEMG acquisition system comprising a matrix based approach for the EMG-sensor array, where the sensors are placed on two flexible PCBs within eight by four grid each and $1 \times 1 \mathrm{~cm}$ grid spacing is presented by Xiong [13]. As a sample rate of $200 \mathrm{~Hz}$ is provided for the 64 channels, the WiFi-standard is used including an access point a Laptop for data processing to match the high demand to the data rate. It is furthermore described that the system costs are estimated to be at around 1000 Euro for a 32 channel version. It has to be noted, that this setup requires additional reference electrodes to be placed somewhere on the patient's corresponding extremities which has to be taken into account when dealing with amputees.

In comparison to the state of the art, the architecture proposed in this paper provides a good tradeoff between the number of acquisition channels and the cost. Moreover the energy autonomy is optimized considering the size and the constraints need for a wearable acquisition board. The system is also suitable to execute aggressive power management for achieving a deep idle power and yet a substantial energy savings during long monitoring by using compressive sampling methods as described in [14].

\section{SYSTEM ARCHITECTURE}

We started from a testbed where Analog EMG signals are sampled and converted to digital data to ease the signal processing, using DAQ cards with on-board AD-converter chips, as illustrated in Fig. 1. The digital data is then sent to a computer or an integrated microprocessor for processing of the signal. This processing stage includes feature extraction and classification. At this point the sampled data is classified to certain action labels. Using these labels the signals for positionand velocity-control are computed and sent to the motors drivers to produce the actual prosthetic hand motion based on the control signals. This control method is capable of recognizing movement of each fingers of the hand, making the hand control easy so that the subject can move the hand as if the patient would do it with a normal hand.

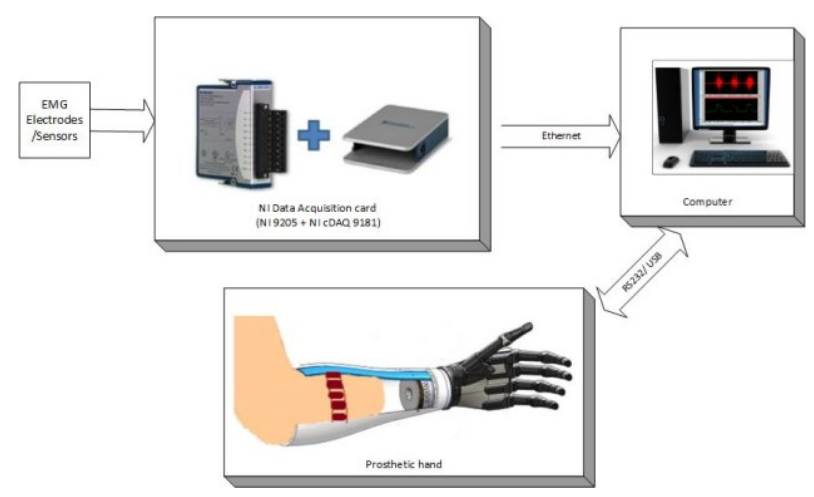

Fig. 1. Schematic block of the preliminary wired acquisition system 
The contribution presented in this paper is to move the acquisition and pre-processing part directly in a board the patient can wear comfortably in the arm close to the set of the sEMG probes. Data communication with suitable bandwidth is provided by Bluetooth wireless protocol and low-power radios as depicted in Fig. 2.

The capability of modern mobile devices can easily substitute a standard desktop workstation used so far for experimental studies. In fact, microprocessors available in those mobile devices have more than just the adequate computational capacity to perform feature extraction, classification and prosthetic hand control. This allows the design of completely novel wearable systems, capable of controlling a prosthetic hand while performing multiple tasks directly commanded by the patient's nervous system in an efficient and cost effective way.

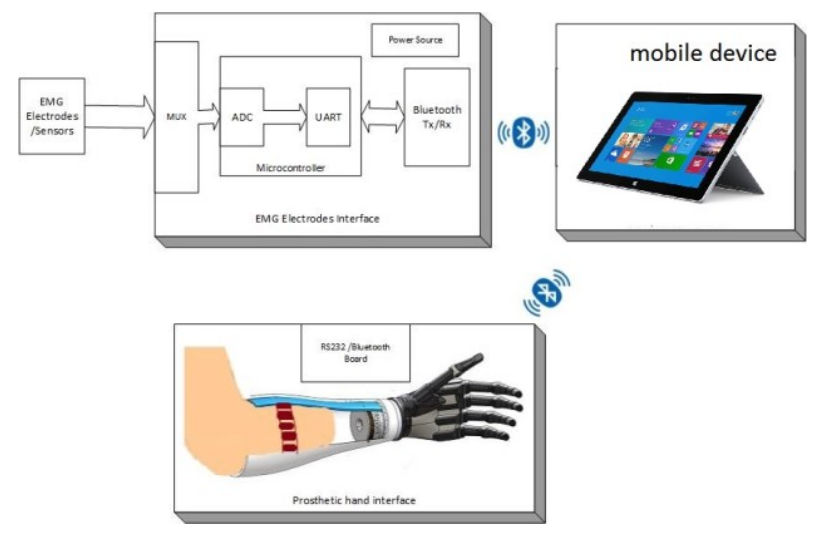

Fig. 2. Functional scheme of the wireless Prosthetic Hand Control with Surface EMG signals

Otto bock 13E200 surface EMG electrodes are used to pick up neuromuscular activity signals. Pattern recognition and machine learning techniques are used to predict the corresponding fingers' movements.

As depicted in Fig. 3, the overall system design consists of three interleaving subsystems: Sensor (EMG-Electrode) interface with pre filtering and acquisition, a mobile processing device (e.g. a small laptop) and the control interface to the Prosthetic hand. The primary purposes of the sensor interface, placed on the patient's remaining forearm stump, is the acquisition of the EMG signals from the incorporated electrodes, the framing of data-packets and the transmission to the mobile device with the help of a Bluetooth connection. Of course, the acquisition system is part of a more complex communication protocol thus it provides means of connection establishment, security and data integrity. Mobile computation capabilities - i.e. the mobile device - are made available by a small Intel Core i5 520M (mobile version) based notebook including at least two Bluetooth interfaces. It interprets and classifies the incoming data-streams from the sEMG probes and transmits control commands to the robotic hand prosthesis. Thus it serves as a central Bluetooth based communication node in between both - the sensor-acquisition node and the prosthesis node. After parsing preprocessing the data into a convenient form for the machine learning program (used for pattern classification), the mobile platform can interact with the prosthetic hand or directly display the analog signal by reconstruction from the digital data. The prosthetic interface handles the received communication data from the computer, i.e. commands generated by the mobile device will be sent to the prosthetic hand using this interface. With respect to the consecutive design considerations for this system, the future aim is a condensed, integrated circuitry providing all necessary components. To achieve this, a detailed analysis of requirements for each one of the components and the possibility of merging the components in future, leading to a considerable reduction of component size was conducted. Of course, a further remarkable aim was to use non expensive components of the shelf (COTS) as far as possible.

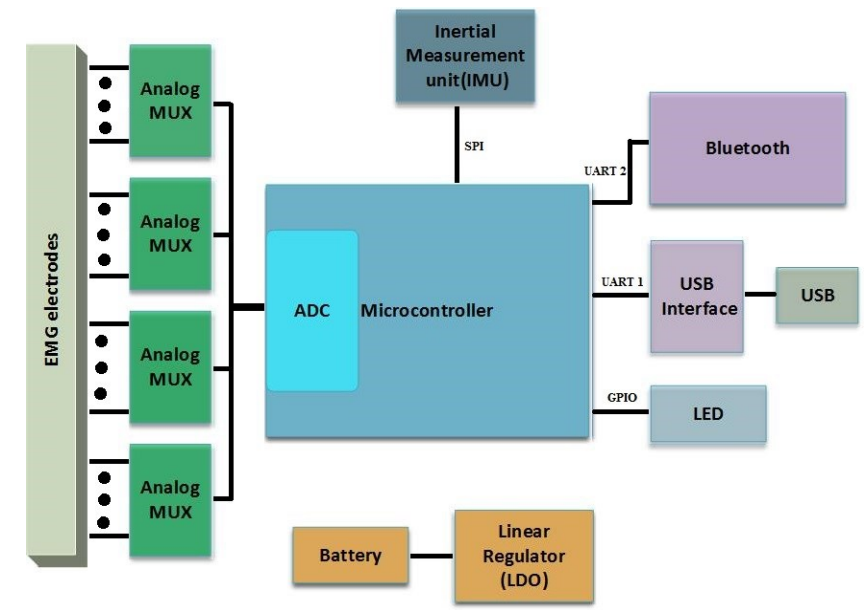

Fig. 3. Schematic block of the wireless communication system based on Bluetooth radio.

In more detail, the designed wireless EMG sensor acquisition system depicted in Fig. 4 incorporates the following features: 32 EMG multiplexed sensor inputs with passive single stage pre-filtering (low pass), a 16 Bit low cost microcontroller (MSP430 derivate with $25 \mathrm{MHz}$ ), an onboard Bluetooth transceiver, an optional USB port for direct PCconnection and debugging, an inertial measurement unit (IMU), several indicator LEDs, and a rechargeable battery. In the current version, the electronics are split, i.e. the controller part including power management and communication can be separated from the analog part. This offers scalability to 64 and more EMG sensors, furthermore, the layout on the analog part can be optimized for best analog signal integrity.

The Bluetooth 2.1 compatible transceiver, a Roving Networks RN-41, provides a low power, bi-directional RF communication link with a $240 \mathrm{kbps}$ data-rate. It is connected to the controller by UART. Also the controller was selected according to its low power capabilities, using less than $10 \mathrm{~mA}$ when operation in full performance mode. The final cost of the wearable multichannel sEMG was required to be below 150 
Euros, excluding the costs for the EMG probes. The prototype developed and tested is illustrated in Fig. 4.

As the power consumption for the analog part is far less than the one of the controller, a lightweight $45 \mathrm{~g}, 2000 \mathrm{mAh}$ LiPo rechargeable battery with the form factor of the board, can keep the acquisition system alive for at least two days running in full power mode. An optimization of the energy management has not been done yet but could increase the mobile capabilities by far.

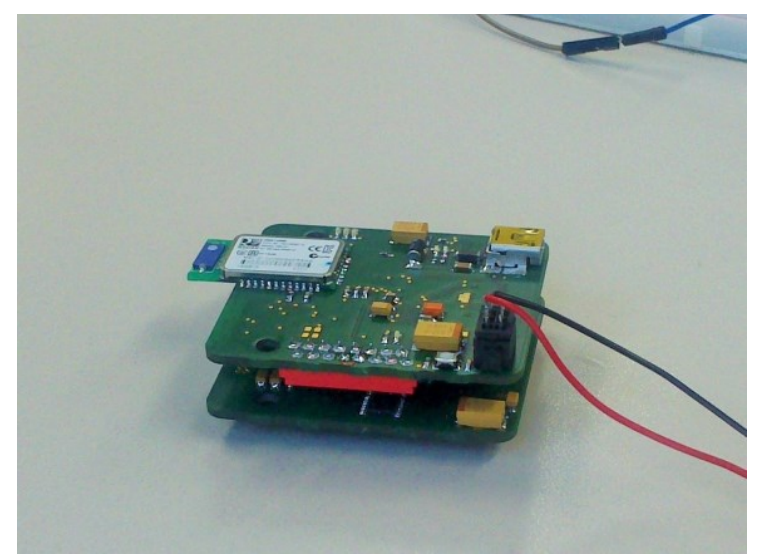

Fig. 4. Prototype of the Low cost mobile Multichannel Surface EMG Acquisition for Prosthetic Hand Control.

\section{A. Otto-Bock sEMG electrodes:}

The Myobock 13E200 EMG electrodes from Ottobock are used to acquire the sEMG signal for the control of the prosthetic hand. These electrodes are firmly placed on the skin to gather the information about the contraction of the muscles underneath. The Myobock electrodes are selected because of some special features than the other electrodes, which include: linear and proportional signal output, highly sensitive amplifiers, high common mode rejection ratio (CMRR) for low frequency which provides a good rejection of electromagnetic interference and high input impedance which can reduce the effect due to the variability of the skin impedance. The Myobock electrodes have frequency bandwidth of around $30 \mathrm{~Hz}$, and can operate with power source of $5 \mathrm{~V}$ to give an output voltage proportional to the level of muscle activation. The electrodes have a 3 pin connector receptacle (Analog output, Power input and ground).

The signal resolution, accuracy, signal range and sampling rate define the quality of the EMG signal. The input impedance of the analog front end system must be as high as possible since the skin-electrode contact impedance is variable from a few $\mathrm{K} \Omega$ to several $\mathrm{M} \Omega$. The quality of the EMG signal is also largely dependent on the resolution, accuracy and sampling rate of the ADC. Present day ADCs used in the EMG systems use 10 to 24bit resolution, however the resolution of ADCs incorporated in most of today's low cost microcontrollers is limited to 12 or 16 bits. Considering the number of the acquired channels and the bandwidth of the sensors, at least ten times oversampling will be considered to improve the output resolution of the data stream. This also enables reduction of aliasing and phase distortion.

\section{B. Acquisition and filter unit}

The used microcontroller MSP430F5529 by Texas Instruments, a 16-bit MCU with built in modules such as ADC, UART and DMA greatly showed the possible reduction of the consumed energy used for conversion and data-transfer. The 12 bit $\mathrm{ADC}$, i.e. one of the core components in the data acquisition system eliminates the need for external ADC chips. The ADC and the UART are configured to work in parallel, thanks to the MCU's DMA capability, which frees the microcontroller's performance for interrupt handling and auxiliary computation, such as digital inline filtering. Combined with the external, passive low pass filters, a precise acquisition process is possible. Due to the separation of the analog part from the computational and communication depended part, a future extensibility to at least 64 input channels is possible with minor configurations. At the moment, eight out of 14 channels are consumed. Further 32 input channels can be added by stacking a second multiplexer module on top of the main board.

\section{Bluetooth communication.}

The Bluetooth communication is realized by a RN-41 Bluetooth Module from Roving Networks. It is available with a small form factor of approximately $3 \mathrm{~cm}^{2}$ including the antenna. It supports the Serial Port Profile (SPP) up to 240 kbps, allowing its host device to appear as a virtual COM port on standard mobile devices. This greatly simplifies the communication protocol, as only the application layer has to be dealt with. Moreover the module is optimized in power consumption (20mA in full transceiver mode) and it requires no external circuitry, further decreases additional power losses. A tailored application layer protocol is used on the elaboration platform to establish a Bluetooth connection with the remote sEMG acquisition board.

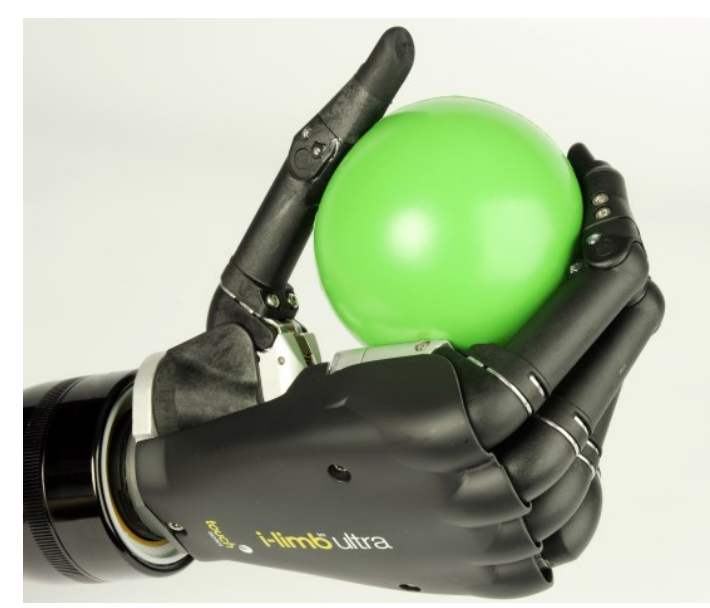

Fig. 5. Picture of the used prosthetic hand. 


\section{Prosthetic Hand Interfacing}

The prosthetic hand, used in this research, is the i-LIMB hand produced by Touch Bionics (see Fig. 5). It is an externally powered, multi-fingered hand with interesting features about controlling and consumption. It is suitable for experimenting on prosthetic control allowing the user to control each one of the four fingers and the motorized rotatable thumb. The control of the hand can be achieved by an UART interface connected to a further Bluetooth adapter (COTS based interface). This connection is established with the second Bluetooth adapter of the mobile device.

\section{E. Machine Learning Methods for sEMG-Based Hand Movement Classification}

The state of the art comprises a lot of machine learning methods that are applied to sEMG signals with promising results [15],[16],[17]. The main blocks of the classification chain needed in the prosthetic hand control consist on i) filtering and pre-processing; ii) segmentation; iii) features extraction and iv) classification.

The signals acquired using by sEMG sensors are usually affected by noise filtering is necessary as part of the preprocessing step. The most critical is the one coupled by the power line available in the environment. Even in batteryoperated systems, the noise induced by cabling in the $50-60 \mathrm{~Hz}$ range can be lead to inferior signal quality, since the useful signal bandwidth correlates with the noise. However, this can be easily addressed exploiting the potential of the on-board microcontroller or using a suitable low power hardware accelerator [18]. Segmentation is fundamental when it is necessary to split streams of sensor data into fragments for post-processing algorithms like features extraction and classification.

In our system, we aim at using methods already well experimented in the wired testbed, as depicted in [19],[20]. Even though the description of the methods are out of the scope of this paper, for the sake of completeness, they are based on SVM models previously built. They clearly show that machine learning-based classification and regression applied to surface EMG achieve good performance, in particular in recognition rate for grasp-type classification. Summarizing, by the combination of a high number of surface electromyography, a significant increases in accuracy can be achieved.

The main challenge in moving toward a low cost and low power wireless prosthetic hand control system is introducing software optimizations in all the steps presented, for reducing the power consumption of the classifier. At the same time, we aim at boosting the classification phase by reducing the delays between EMG stimuli and actuation.

\section{EXPERIMENTAL SETUP}

We performed several experiments to assess the signal quality of the data received by comparing the data received with a standard wired, high quality data acquisition card by National Instruments. The same EMG stimuli are fed to each one of the two sets of sEMG sensor setups, i.e. the wireless and the wired version (see Fig. 6).

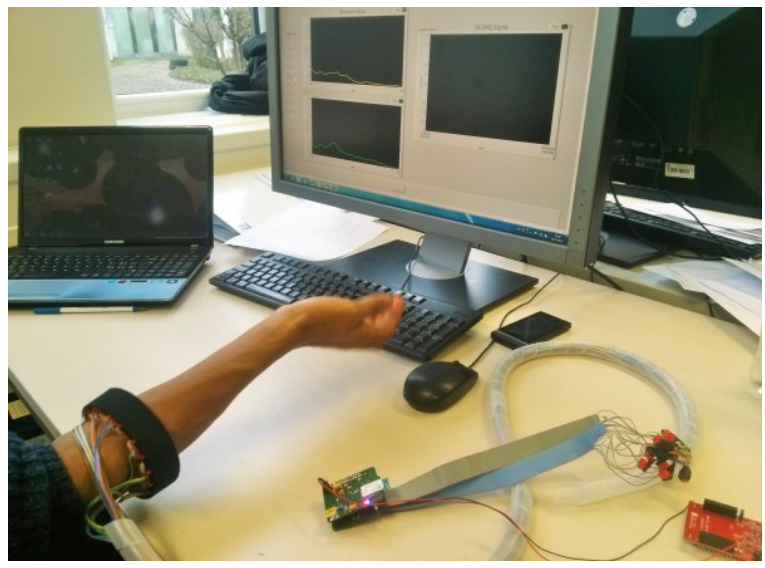

Fig. 6. Phase of the experimental measurements of the proposed system.

For both setups we used a standard PC workstation of similar performance to receive and process the acquired data, as the focus had been on the wireless acquisition node and not on demonstration of mobile capability. A LabVIEW application was used to display and compare the accuracy online, the experimental data is shown in Fig. 7.
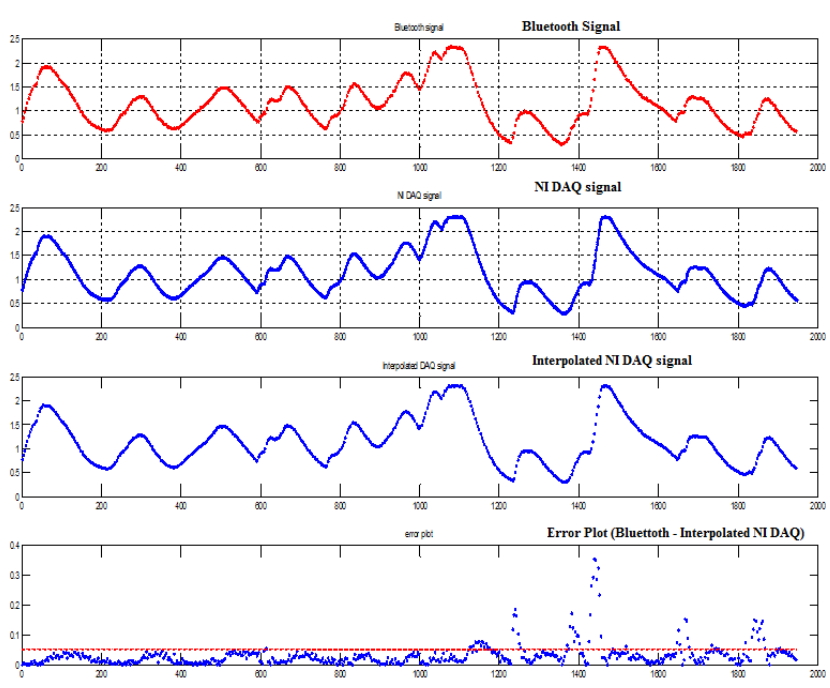

Fig. 7. Experimental comparison between a traditional DAQ system and the presented sEMG wireless acquisition board.

A waveform graph is used to display a single set of samples received from the computers serial port buffer. Lab-View provides an easy access to the computers serial ports through the use of the integrated VISA interface, including the Bluetooth dongles that emulate virtual COM-ports in the serial port profile (SPP). To visualize the EMG signals sent from the acquisition board, we parse the data byte-wise. Matlab is then used for quantitative analysis on the samples. The number of 
samples acquired by the wireless acquisition module and the National Instruments DAQ card may differ, also slight jitter is possible due to the lacking synchronization of the both signal sources; consequently, interpolation techniques must be used to compare samples from the wireless acquisition system with the National Instruments DAQ system.

As depicted by the results in Fig. 7, the mean square error is quite below $50 \mathrm{mV}$ with direct differential comparison. Slight oscillations and big overshoots are assumed to be a result of jitters between both source signals. This result is considered very promising considering the priority set requirements for sEMG acquisition systems.

The current consumption of the developed wireless sEMG acquisition board has been measured to be at $32 \mathrm{~mA}$, which results in a maximum of two and a half days of continuous, independent operation together with the proposed $2000 \mathrm{mAh}$ rechargeable battery. This is well beyond the interval of 24 hours usually considered a critical threshold for pushing a mobile device onto the market. Concerning the production price, the developed wireless acquisition system showed with approximately 60 Euros - to be far less expensive than the required target price, with the prototype multilayer board being the most expensive part.

\section{CONCLUSION}

In this paper, the development of a fully wireless, low cost sEMG acquisition system has been presented. The EMG signals are acquired from the electrodes attached on the patient's remaining forearm stump using Otto-bock 13E200 EMG electrodes. This system, based on the MSP430F5529 microcontroller, converts the analog signals into a digital data stream that is sent to a mobile processing device by Bluetooth. Experimental analysis shows that the output signals of the wireless acquisition system correlates to an industrial data acquisition board based on National Instruments NI-DAQ, the maximum mean square error is below $50 \mathrm{mV}$. The aimed pricing for the system could well be undercut by more than the half. The systems scalability and the possibility to draw distributed subsystems together is a promising strategy for further steps.

\section{ACKNOWLEDGMENT}

The work presented in this paper was supported by the project GreenDataNet, funded by the EU 7th Framework Programme (grant n.609000).

\section{REFERENCES}

[1] Castellini, C. \& van der Smagt, P. Surface EMG in advanced hand prosthetics Biological Cybernetics, 2009, 100, 35-47

[2] Guanglin Li: Electromyography Pattern-Recognition-Based Control of Power Multifunctional Upper-Limb Prostheses, 2007
[3] Kuiken, T.; Li, G.; Lock, B.; Lipschutz, R.; Miller, L.; Stubblefield, K. \& Englehart, K: Targeted muscle reinnervation for real-time myoelectric control of multifunction artificial arms, JAMA., vol. 301, pp. 619-628., 2009

[4] K.R. Wheeler, C.C. Jorgensen, Gestures as input: neuroelectric joysticks and keyboards, Pervasive Computing 2 (April-June (2)) (2003) 56-61.

[5] M.A. Oskoei, H. Hu, Myoelectric control systems-a survey, Biomedical Signal Processing and Control 2 (October (4)) (2007) 275294.

[6] Xun Chen, Z. Jane Wang, Pattern recognition of number gestures based on a wireless surface EMG system, Biomedical Signal Processing and Control, Volume 8, Issue 2, March 2013, Pages 184-192

[7] Wang C.S., Methodology Report - Design of a 32-channel EEG System for Brain Control Interface Applications, Hindawi Journal of Biomedicine and Biotechnology, Volume 2012, Article ID 274939

[8] Bortolotti, D., Mamaghanian, H., Bartolini, A., Ashouei, M., Stuijt, J., Atienza, D., Vandergheynst, P., Benini, L., Approximate compressed sensing: Ultra-low power biosignal processing via aggressive voltage scaling on a hybrid memory multi-core processor, (2014) Proceedings of the International Symposium on Low Power Electronics and Design, pp. 45-50.

[9] L.M. Vaca Benitez, M. Tabie, N. Will, S. Schmidt, M. Jordan, E.A. Kirchner, Exoskeleton Technology in Rehabilitation: Towards an EMGBased Orthosis System for Upper Limb Neuromotor Rehabilitation, Hindawi Journal of Robotics, Volume 2013, Article ID 610589

[10] BRAIN PRODUCTS GmbH, BrainAmp ExG mR Technical Specifications, www.brainproducts.com, 2015

[11] BRAIN PRODUCTS GmbH, PowerPack Technical Specifications, www.brainproducts.com, 2015

[12] S.S. Lee, K.-Y. Shin, J.H. Mun, Development of a Portable and Wireless Surface EMG, Key Engineering Materials Vols 321-223 (2006), pp. 1107-1110

[13] Q. Xiong, Design of an Advanced Portable System for high Density Surface EMG Recording with Wireless Control of Signal Quality. Politecnico di Torino, http://porto.polito.it/2592680, March 2015

[14] Caione, C., Brunelli, D., Benini, L., Compressive sensing optimization for signal ensembles in WSNs, (2014) IEEE Transactions on Industrial Informatics, 10 (1), art. no. 6523111, pp. 382-392.

[15] B. Karlik, M. Osman Tokhi, M. Alci, A fuzzy clustering neural network architecture for multifunction upper-limb prosthesis, IEEE Transactions on Biomedical Engineering 50 (11) (2003) 1255-1261.

[16] Jun-Uk Chu, Inhyuk Moon, Mu-Seong Mun, A real-time EMG pattern recognition system based on linear-nonlinear feature projection for a multifunction myoelectric hand, IEEE Transactions on Biomedical Engineering 53 (11) (2006) 2232-2239.

[17] K.S. Kim, H.H. Choi, C.S. Moon, C.W. Mun, Comparison of k-nearest neighbor, quadratic discriminant and linear discriminant analysis in classification of electromyogram signals based on the wrist-motion directions, Current Applied Physics 11 (3) (2011) 740-745.

[18] Benatti, S.; Milosevic, B.; Casamassima, F.; Schonle, P.; Bunjaku, P.; Fateh, S.; Huang, Q.; Benini, L., "EMG-based hand gesture recognition with flexible analog front end," Biomedical Circuits and Systems Conference (BioCAS), 2014 IEEE, vol., no., pp.57,60, 22-24 Oct. 2014

[19] Gijsberts, A.; Atzori, M.; Castellini, C.; Muller, H.; Caputo, B., "Movement Error Rate for Evaluation of Machine Learning Methods for sEMG-Based Hand Movement Classification," Neural Systems and Rehabilitation Engineering, IEEE Transactions on , vol.22, no.4, pp. 735,744 , July 2014

[20] Tommasi, T.; Orabona, F.; Castellini, C.; Caputo, B., "Improving Control of Dexterous Hand Prostheses Using Adaptive Learning," Robotics, IEEE Transactions on, vol.29, no.1, pp.207,219, Feb. 2013. 